Original Article

\title{
Effects on short term outcome of non-invasive ventilation use in the emergency department to treat patients with acute heart failure: A propensity score-based analysis of the EAHFE Registry
}

Òscar Miróa,b,c,*, Gemma Martínez $^{\mathrm{a}}$, Josep Masip ${ }^{\mathrm{c}, \mathrm{d}, \mathrm{e}}$, Víctor Gil ${ }^{\mathrm{a}}$, Francisco Javier Martín-Sánchez ${ }^{\mathrm{f}}$, Pere Llorens ${ }^{\mathrm{g}}{ }^{\text {, Pablo Herrero-Puente }}{ }^{\mathrm{h}}$, Carolina Sánchez ${ }^{\mathrm{a}}$, Fernando Richard ${ }^{\mathrm{i}}$, Javier Lucas-Invernón ${ }^{\mathrm{j}}$, José Manuel Garrido ${ }^{\mathrm{k}}$, Alexandre Mebazaa ${ }^{\mathrm{c}, \mathrm{l}}$, José Ríos $^{\mathrm{m}}$, W. Frank Peacock ${ }^{\mathrm{c}, \mathrm{n}}$, Judd E. Hollander ${ }^{\mathrm{o}}$, Javier Jacob ${ }^{\mathrm{p}}$, on behalf of the ICA-SEMES Research Group Researchers

${ }^{a}$ Emergency Department, Hospital Clínic, Barcelona, Spain

${ }^{\mathrm{b}}$ Medical School, University of Barcelona, Spain

c The GREAT Network, Italy

d Cardiology Department, Hospital Sanitas CIMA Barcelona, Spain

e Hospital de St Joan Despí Moisès Broggi, University of Barcelona, Spain

${ }^{\mathrm{f}}$ Emergency Department, Hospital Clínico San Carlos, Universidad Complutense de Madrid, Madrid, Spain

${ }^{g}$ Emergency Department, Home Hospitalization and Short Stay Unit, Hospital General de Alicante, Alicante, Spain

h Emergency Department, Hospital Universitario Central de Asturias, Oviedo, Spain

${ }^{\mathrm{i}}$ Emergency Department, Hospital Universitario de Burgos, Spain

${ }^{\mathrm{j}}$ Emergency Department, Hospital General Universitario de Albacete, Spain

${ }^{\mathbf{k}}$ Hospital Universitario Vírgen Macarena, Sevilla, Spain

${ }^{1}$ Department of Anesthesiology and Critical Care Medicine, Hospital Lariboisière, Université Paris Diderot, Paris, France

${ }^{\mathbf{m}}$ Laboratory of Biostatistics \& Epidemiology, Universitat Autònoma de Barcelona, Medical Statistics Core Facility, IDIBAPS, Hospital Clinic, Barcelona, Spain

${ }^{n}$ Department of Emergency Medicine, Baylor College of Medicine, Houston, TX, USA

o Department of Emergency Medicine, Sidney Kimmel Medical College at Thomas Jefferson University, Philadelphia, PA, USA

P Emergency Department, Hospital Universitari de Bellvitge, L'Hospitalet de Llobregat, Barcelona, Spain

\section{A R T I C L E I N F O}

\section{Keywords:}

Non-invasive ventilation

Heart failure

Acute heart failure

Emergency department

Outcome

\begin{abstract}
A B S T R A C T
Objective: To assess the effects of non-invasive ventilation (NIV) in emergency department (ED) patients with acute heart failure (AHF) on short term outcomes.

Methods: Patients from the EAHFE Registry (a multicenter, observational, multipurpose, cohort-designed database including consecutive AHF patients in 41 Spanish EDs) were grouped based on NIV treatment (NIV + and NIV-groups). Using propensity score (PS) methodology, we identified two subgroups of patients matched by 38 covariates and compared regarding 30-day survival (primary outcome). Interaction was investigated for age, sex, ischemic cardiomyopathy, chronic obstructive pulmonary disease, AHF precipitated by an acute coronary syndrome (ACS), AHF classified as hypertensive or acute pulmonary edema (APE), and systolic blood pressure (SBP). Secondary outcomes were intensive care unit (ICU) admission; mechanical ventilation; in-hospital, 3-day and 7-day mortality; and prolonged hospitalization ( $>7$ days).

Results: Of 11,152 patients from the EAHFE (age (SD): 80 (10) years; 55.5\% women), 718 (6.4\%) were NIV+ and had a higher 30 -day mortality $(\mathrm{HR}=2.229 ; 95 \% \mathrm{CI}=1.861-2.670)(\mathrm{p}<0.001)$. PS matching provided 2 groups of 490 patients each with no significant differences in 30-day mortality $(\mathrm{HR}=1.239$; $95 \% \mathrm{CI}=0.905-1.696)(\mathrm{p}=0.182)$. Interaction analysis suggested a worse effect of NIV on elderly patients ( $>85$ years, $\mathrm{p}<0.001$ ), AHF associated with ACS ( $\mathrm{p}=0.045)$, and SBP $<100 \mathrm{mmHg}(\mathrm{p}<0.001)$. No significant differences were found in the secondary endpoints except for more prolonged hospitalizations in NIV + patients $(\mathrm{OR}=1.445 ; 95 \% \mathrm{CI}=1.122-1.862)(\mathrm{p}=0.004)$.
\end{abstract}

\footnotetext{
* Corresponding author at: Emergency Department, Hospital Clínic, Villarroel 170, 08036 Barcelona, Catalonia, Spain.

E-mail address: omiro@clinic.cat (Ò. Miró).

Marta Fuentes ${ }^{q}$ Cristina Gil ${ }^{q}$ Héctor Alonso ${ }^{r}$ Pablo Garmila ${ }^{r}$ Esther Rodríguez Adrada ${ }^{s}$ Rosa Escoda ${ }^{t}$ Carolina Xipell ${ }^{t}$ lank $>$
} 
Conclusion: The use of NIV to treat AHF in ED is not associated with improved mortality outcomes and should be cautious in old patients and those with ACS and hypotension.

\section{Introduction}

Non-invasive ventilation (NIV) for ventilatory support is commonly used in several types of respiratory failure. Patients with acute heart failure (AHF) may receive NIV, especially those presenting with acute pulmonary edema (APE). A meta-analysis of 15 clinical trials published between 1988 and 2005 concluded that NIV reduced the need for intubation and mortality in patients with APE and that there were no differences between the two common NIV modalities: continuous positive airway pressure (CPAP) and bi-level positive airway pressure (BIPAP) [1]. However, most studies had a small number of patients and, overall, only 389 patients were treated with NIV in these 15 studies. In one large evaluation from the retrospective ADHERE heart failure database reported in 2008, Tallman et al. compared the outcomes of 2430 patients with severe respiratory distress receiving NIV versus those with immediate endotracheal intubation (ETI). Regardless of the ultimate need for ETI, patients receiving NIV fared no worse than the cohort receiving immediate ETI, suggesting that, compared to the alternative of ETI, NIV may be a reasonable intervention in AHF patients presenting with severe respiratory distress [2].

Nonetheless, recent studies have introduced doubt about the real benefit of NIV in terms of improving short-term mortality. The $3 \mathrm{CPO}$ study by Gray et al. [3], one of the largest randomized controlled trials (RCT) in this setting [4], reached conclusions different from those of the aforementioned meta-analysis and generated a great deal of controversy. These authors randomized 1069 patients with APE to receive NIV or standard oxygen therapy and found that although NIV induced a more rapid improvement in respiratory distress and metabolic disturbance than standard oxygen therapy, it had no effect on 7-day mortality. Finally, a more recent review has highlighted that, although hospital mortality seems to be reduced by NIV, the evidence to date on the potential benefit of NIV in reducing mortality is entirely derived from small trials, and further large-scale trials are needed [5].

Unfortunately, some of the RCT data available on the outcomes of AHF patients in whom NIV is used are from the well-controlled setting of an intensive care unit (ICU) and are not well represented from the less controlled emergency department (ED) environment. The challenges of interpreting non-ED data applied to the ED population may increase the risk of misapplication. The reasons for this may be the result of greater variation in the population, a higher likelihood of misdiagnosis, a larger range in illness severity, and that NIV may be started in the ED (and even by prehospital emergency medical systems -EMS-) and are often removed in the ED itself due to the fact that NIV can improve AHF patient's clinical status in a few hours [6-8]. Moreover, the inclusion and exclusion criteria that a RCT imposes on the final sample of patients selected for analysis excludes a large portion of patients, often surpassing $80 \%$ [8]. Therefore, although there is no doubt that for patients able to tolerate NIV the improvement of symptoms is greater and faster, the effects of NIV on short-term mortality are still controversial when it is used in the ED setting in real world conditions. For this reason, the objective of the present study was to assess the effects on short-term outcome of the use of NIV in the ED setting to treat patients diagnosed with AHF.

\section{Patients and methods}

\subsection{Study setting}

The present study was carried out using the patients included in the EAHFE (Epidemiology of Acute Heart Failure in Emergency
Department) Registry. This is a multicenter, observational, multipurpose, cohort-designed database that includes consecutive patients diagnosed with AHF in 41 Spanish EDs (approximately 7\% of EDs of the Spanish Public Health Care System) including both university and community hospitals from all areas of the country. The characteristics of the EAHFE Registry have been published elsewhere [9-11]. For the present study, we used Phases 3, 4, and 5 EAHFE Registry patients. These phases were respectively carried out in 2011 ( 2 months of recruitment, 25 participating EDs, $\mathrm{n}=3414$ ), 2014 (2 months, 27 EDs, $\mathrm{n}=3233$ ), and 2016 ( 2 months, 30 EDs, $\mathrm{n}=4715$ ), with similar recruitment dynamics along the 3 periods. Briefly, patients were included by the attending emergency physicians, all of whom received specific instructions regarding the study protocol during a meeting held in every ED the week before each recruitment period. All cases were doublechecked by the principal investigator of each center prior to inclusion into the database in order to ascertain that patients met the clinical diagnostic criteria of AHF. In addition, when possible and available, the diagnosis was confirmed by natriuretic peptide determination or echocardiography, following the European Society of Cardiology criteria [12], during ED or hospitalization stay (approximately $92 \%$ of cases). Patients with clinical diagnostic criteria, but without echocardiographic or natriuretic peptide determinations, were accepted in order to have a cohort similar to that observed in the routine emergency medicine practice. Final diagnostic adjudication was completed by the principal investigator of each center. All principal investigators were provided with a dictionary of terms to ensure common definitions (available in Supplementary Table 1). The only exclusion criterion in the EAHFE Registry was patients with a primary diagnosis of ST-elevation myocardial infarction (STEMI) who concurrently developed AHF (approximately $3 \%$ of AHF cases), as these patients follow a very different clinical pathway that frequently by-passes the ED.

\subsection{Variables analyzed}

We collected 38 variables potentially related to prognosis in every patient included in the study. This included demographics, clinical history, presentation, and treatments based on the authors' consensus after review of previous literature [13-15]. These covariates were reported in specific case report forms during ED attendance (see Supplementary Table 1). Interventions, treatments and patient allocation (hospital admission or discharge) were entirely based on the decisions of the attending ED physician. Subsequent follow-up by telephone contact and consultation of medical records was performed between days 31 and 90 after ED attendance in order to detect all-cause death. The whole EAHFE Registry protocol was approved by a single central Ethical Committee at the Hospital Universitario Central de Asturias (Oviedo, Spain), with reference numbers 49/2010, 69/2011, 166/13, and $160 / 15$. Due to the non-interventional design of the study, Spanish legislation allows the remaining centers participating in a multicenter study to include patients with central Ethical Committee approval provided that their local Ethical Committees are duly informed about their participation. All patients gave informed consent to be included in the EAHFE Registry and to be contacted for follow-up. Around $2 \%$ of patients fulfilling the inclusion and exclusion criteria refused to participate and did not sign informed consent.

\subsection{Statistical analysis}

The classification variable was the use of NIV (NIV + and NIVgroups, respectively) in the ED. Thirty-eight covariates were evaluated 
by the chi-square or Student's t-test (or Mann-Whitney U test when appropriate) depending on whether they were qualitative or quantitative variables, respectively. We used propensity score (PS) matching methodology to obtain two comparable cohorts in the NIV + and NIVgroups. A PS was estimated for each of the patients [16] using multivariate logistic regression and determined the probability that participants would receive NIV based on their individual characteristics (covariates). For this purpose, we constructed a multivariable model including the independent variables that significantly differed between groups in the univariable analysis (defined as a $\mathrm{p}<0.10$ ) in order to obtain pairs of patients with comparable epidemiological, baseline, clinical and therapeutic characteristics. It has been demonstrated that this method provides accurate estimates of the effect of a drug in observational settings by minimizing confounding factors by indication [17], and it has been proposed as a solution to overcome immortal time bias (from the patient's entrance into the cohort to study drug intake) in pharmacologic and epidemiologic studies [18]. Finally, patients were paired (1:1) according to the nearest neighbour approach within a calliper width of 0.01 . To assess the balance of covariates between groups after PS matching, $<10 \%$ of relative difference of means or proportions was considered to support the assumption of balance between the NIV+ and NIV- groups.

The primary endpoint was 30-day all-cause mortality, which was determined by survival tables using the Kaplan-Meier method, and the log-rank test was used for comparison between the NIV + and NIVgroups. The risk of all-cause death was calculated by estimation of hazard ratios (HR) with the $95 \%$ confidence interval $(95 \% \mathrm{CI})$ for patients of the NIV + group compared to those of the NIV-group using the Cox regression model, and was initially calculated for the entire cohort (unadjusted) and then for the PS-matched groups. The subgroups of NIV + patients according to the modality of NIV used (CPAP or BIPAP) were also analyzed. We also planned a priori to investigate interaction for the primary endpoint (30-day mortality) in the PS-matched patients for the following groups: elderly patients (cut-off: 85 years), sex, ischemic cardiomyopathy or chronic obstructive pulmonary disease (COPD) as comorbidities, AHF precipitated by an acute coronary syndrome (ACS), AHF classified as hypertensive or APE forms, and low systolic blood pressure (SBP) (cut-off: $100 \mathrm{mmHg}$ ) using Cox models. The secondary endpoints analyzed in the PS-matched patients were need for ICU admission, need for OTI and mechanical ventilation, 3day, 7-day and in-hospital mortality, and prolonged hospitalization (defined as $>7$ days from ED admission to final hospital discharge). All secondary endpoints were expressed by odds ratios (OR) and 95\%CI for the patients of the NIV + group compared to the NIV- group. All statistical tests were performed with a two-sided type I error of 5\%, and we used statistical software (SPSS v 19.0) for all the calculations.

\section{Results}

Of the 11,380 patients consecutively included recruitment phases 3 , 4 and 5 of the EAHFE Registry, 11,152 were included in the NIV-AHF Study (Fig. 1). The mean age was 80 (10) years, and 55.5\% were women. This cohort had a high number of concomitant comorbidities, and most presented some degree of limitation at baseline: $75.6 \%$ were in NYHA class II or higher, and $58.3 \%$ had at least moderate dependence (Barthel index of 90 or less points). The remaining clinical data are shown in Table 1.

NIV was used in the ED in 718 patients (6.4\%); CPAP in 317 (44,2\%), BiPAP in 380 (52.9\%), and both were attempted in 21 (2.9\%). $\mathrm{NIV}+$ patients differed from NIV- in 22 of the 36 variables analyzed (Table 1): they were younger; more frequently had hypertension, diabetes mellitus, chronic kidney disease, cerebrovascular disease, peripheral vascular disease and COPD, and less frequently atrial fibrillation; had worse functional status at baseline (assessed by the Barthel index); were more frequently receiving chronic treatment with nitrates and amiodarone, and less frequently with digoxin; had a higher SBP and heart rate, and lower pulse oximetry at ED arrival; has higher glycemia and creatinine values during the acute decompensation; were more frequently treated with intravenous diuretics, nitrates, morphine, inotropics/vasopressors at ED; and were more frequently hospitalized.

With respect to the primary endpoint of 30-day all-cause mortality, 1081 patients (9.7\%) died during the 30-day follow up: 135 (18.8\%) in the NIV+ versus $946(9.1 \%)$ in the NIV- group. The HR for the NIV+ group in the unadjusted analysis was 2.229 (95\% CI: 1.861-2.670; $\mathrm{p}<0.001$ ); with very similar curves and HRs when CPAP and BiPAP modalities were analyzed separately (Fig. 2).

Propensity score matching for the 22 variables with significant differences between groups provided 490 pairs of patients matched by the probability of being treated with NIV. The median PSs for the NIV + and NIV- patients were 0.160 (p25-75: 0.055-0.404) and 0.161 (P25-75: 0.005-0.404) ( $\mathrm{p}=.998)$, respectively. These groups did not significantly differ in any of the 36 independent variables, and in only three variables (previous history of ischemic cardiomyopathy, and treatment with an aldosterone-receptor blocker or amiodarone) did the relative difference between both groups exceed 10\% (Table 1).

After PS matching, there was no difference in the primary endpoint between PS-matched groups, and the HR for NIV + was HR $=1.239$ (95\% CI: 0.905-1.696; $\mathrm{p}=0.182$ ), with very similar results for CPAP and BiPAP (Fig. 3). The pre-planned stratified analysis showed an interaction with elderly age ( $>85$ years), AHF associated with ACS, and with a low SBP $(<100 \mathrm{mmHg})$, in all cases having poorer prognosis if NIV was used versus if it was not used (Fig. 4).

The analysis of the secondary endpoints did not uncover significant differences in terms of the need for intensive care admission after ED management, need for ETI and mechanical ventilation, and 3-day, 7day or in-hospital mortality. However, patients in the NIV + group more frequently required prolonged hospitalization (OR: 1.445; CI 95\%: 1.122-1.862; $\mathrm{p}=0.004$ ) (Table 2).

\section{Discussion}

The present study investigated the impact of ED use of NIV to treat AHF patients in real world conditions and its association with shortterm patient outcomes. Except in very eldery subjects, hypotensive

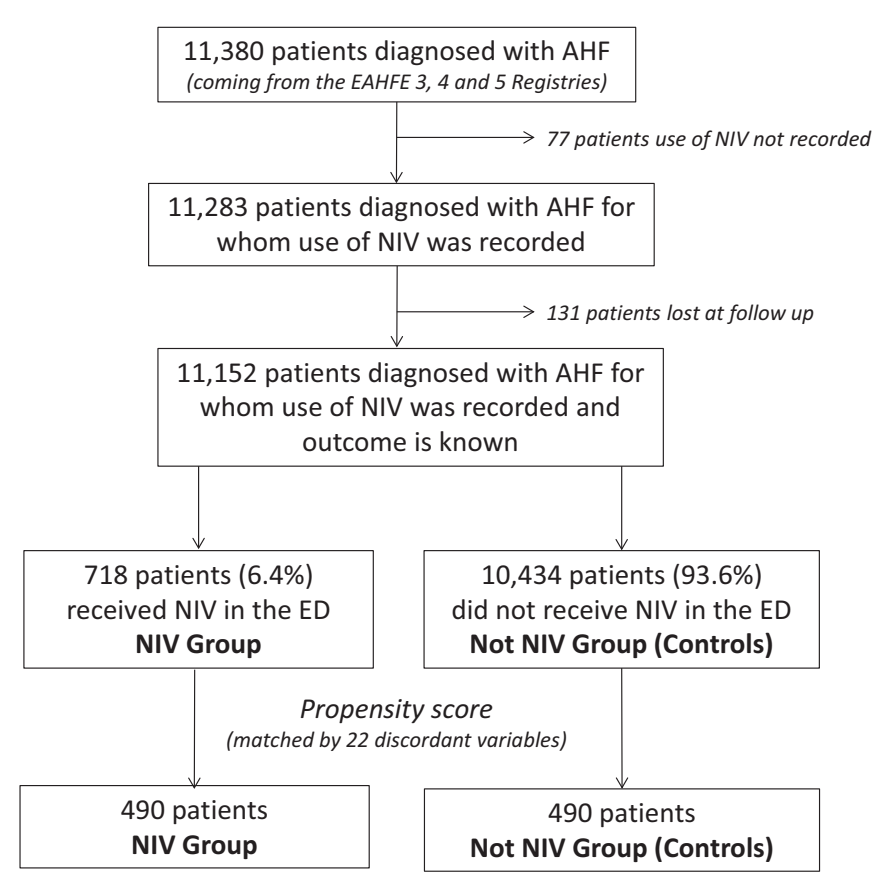

Fig. 1. Flow chart of the patients studied.

NIV: non-invasive ventilation; COPD: chronic obstructive pulmonary disease; ED: emergency department. 
Table 1

Characteristics of patients included in the NIV-AHF Study and comparison between the two treatment groups.

\begin{tabular}{|c|c|c|c|c|c|c|c|c|c|}
\hline & \multicolumn{5}{|c|}{ Groups coming from the whole cohort } & \multicolumn{4}{|c|}{ Propensity-score matched groups } \\
\hline & Total & $\begin{array}{l}\text { Missing } \\
\text { values }\end{array}$ & $\mathrm{NIV}+$ & NIV- & \multirow[t]{2}{*}{$\mathrm{p}$ value } & $\mathrm{NIV}+$ & NIV- & \multirow[t]{2}{*}{$\mathrm{p}$ value } & \multirow[t]{2}{*}{$\mathrm{RD}^{\mathrm{a}}(\%)$} \\
\hline & $\begin{array}{l}\mathrm{N}=11,152(\mathrm{n} \\
[\%])\end{array}$ & $(\mathrm{n}[\%])$ & $\begin{array}{l}\mathrm{N}=718(\mathrm{n} \\
[\%])\end{array}$ & $\begin{array}{l}\mathrm{N}=10,434(\mathrm{n} \\
[\%])\end{array}$ & & $\begin{array}{l}\mathrm{N}=490(\mathrm{n} \\
[\%])\end{array}$ & $\begin{array}{l}\mathrm{N}=490(\mathrm{n} \\
[\%])\end{array}$ & & \\
\hline \multicolumn{10}{|l|}{ Demographic data } \\
\hline Age (years) (mean (SD)) & $80(10)$ & $16(0.1)$ & $79(9)$ & $80(10)$ & $<0.001$ & $79(10)$ & $79(11)$ & 0.510 & 0 \\
\hline Female & $6167(55.5)$ & $44(0.4)$ & $392(54.7)$ & $5575(55.6)$ & 0.697 & $268(54.8)$ & $273(55.8)$ & 0.797 & -1.8 \\
\hline \multicolumn{10}{|l|}{ Comorbidities } \\
\hline Hypertension & $9361(84)$ & $11(0.1)$ & $626(87.2)$ & $8735(83.8)$ & 0.019 & $425(86.7)$ & $428(87.3)$ & 0.849 & -0.6 \\
\hline Diabetes mellitus & $4678(42)$ & $12(0.1)$ & $389(54.2)$ & $4289(41.2)$ & $<0.001$ & $258(52.7)$ & $273(55.7)$ & 0.369 & -5.4 \\
\hline Dyslipidemia & $4959(44.5)$ & $12(0.1)$ & $336(46.8)$ & $4623(44.4)$ & 0.218 & $234(47.8)$ & $241(49.2)$ & 0.701 & -2.8 \\
\hline Ischemic heart disease & $3210(28.8)$ & $11(0.1)$ & $216(30.1)$ & $2994(28.7)$ & 0.462 & $136(27.8)$ & $164(33.5)$ & 0.061 & -13.0 \\
\hline Chronic kidney failure & $2978(26.7)$ & $10(0.1)$ & $225(31.4)$ & $2753(26.4)$ & 0.004 & $165(33.7)$ & $165(33.7)$ & 1.000 & 0 \\
\hline Cerebrovascular disease & $1468(13.2)$ & $12(0.1)$ & $118(16.4)$ & $1350(13)$ & 0.009 & $75(15.3)$ & $72(14.7)$ & 0.858 & +4.1 \\
\hline Atrial fibrillation & $5516(49.5)$ & $9(0.1)$ & 279 (38.9) & $5237(50.2)$ & $<0.001$ & $207(42.2)$ & $204(41.6)$ & 0.897 & +1.4 \\
\hline Peripheral arterial disease & $1045(9.4)$ & $13(0.1)$ & $92(12.8)$ & $953(9.1)$ & 0.001 & $57(11.6)$ & $59(12.0)$ & 0.921 & -3.3 \\
\hline Heart valve disease & $2989(26.8)$ & $11(0.1)$ & $181(25.2)$ & $2808(26.9)$ & 0.332 & $124(25.3)$ & $120(24.5)$ & 0.825 & +3.3 \\
\hline COPD & $2682(24.1)$ & $12(0.1)$ & $226(31.5)$ & $2456(23.6)$ & $<0.001$ & $160(32.7)$ & $159(32.4)$ & 1.000 & +0.9 \\
\hline Dementia & $1430(12.8)$ & $11(0.1)$ & $105(14.6)$ & $1325(12.7)$ & 0.150 & $67(13.7)$ & $67(13.7)$ & 1.000 & 0 \\
\hline Prior episode of heart failure & $6396(57.8)$ & $90(0.8)$ & $431(60.1)$ & $5965(57.7)$ & 0.213 & $299(61.0)$ & $284(58.0)$ & 0.362 & +5.2 \\
\hline \multicolumn{10}{|l|}{ Baseline status } \\
\hline $\begin{array}{l}\text { Barthel Index (points) (mean } \\
\text { (SD)) }\end{array}$ & $79(24)$ & $1360(12.2)$ & $74(25)$ & $79(24)$ & $<0.001$ & $73(26)$ & $75(28)$ & 0.436 & -2.7 \\
\hline NYHA class II or higher & $8411(75.6)$ & $690(6.2)$ & $551(80.7)$ & $7360(75.3)$ & $<0.001$ & $388(80.3)$ & $372(78.3)$ & 0.327 & +2.6 \\
\hline LVEF (\%) (mean (SD)) & $51(15)$ & $4995(44.8)$ & $51(16)$ & $51(15)$ & 0.815 & $51(16.3)$ & $49(15.6)$ & 0.088 & +4.1 \\
\hline \multicolumn{10}{|l|}{ Chronic treatments } \\
\hline Diuretics & $8095(74.6)$ & $306(2.7)$ & $525(74.5)$ & $7570(74.6)$ & 0.951 & $363(71.1)$ & $349(71.2)$ & 0.325 & -0.1 \\
\hline ACE inhibitor or ARB & $6346(57.1)$ & $31(0.3)$ & $415(58)$ & $5931(57)$ & 0.644 & $281(57.3)$ & $302(61.6)$ & 0.193 & -7.0 \\
\hline Beta-blocker & $4613(42.5)$ & $309(2.8)$ & $280(39.8)$ & $4333(42.7)$ & 0.134 & $198(38.6)$ & $209(42.7)$ & 0.217 & -9.4 \\
\hline Aldosterone-receptor blocker & $1812(16.7)$ & $305(2.7)$ & $126(17.9)$ & $1686(16.6)$ & 0.420 & $84(17.1)$ & $70(14.3)$ & 0.254 & +19.6 \\
\hline Nitrates & $1857(17.1)$ & $307(2.8)$ & $145(20.6)$ & $1712(16.9)$ & 0.014 & $100(20.4)$ & $107(21.8)$ & 0.639 & -6.4 \\
\hline Amiodarone & $661(6.1)$ & $310(2.8)$ & $56(7.9)$ & $605(6)$ & 0.042 & $35(7.1)$ & $40(8.2)$ & 0.631 & -13.4 \\
\hline Digoxin & $1637(15.1)$ & $313(2.8)$ & $72(10.2)$ & $1565(15.4)$ & $<0.001$ & $52(10.6)$ & $49(10.0)$ & 0.834 & +6.0 \\
\hline \multicolumn{10}{|l|}{ Vitals at ED during acute episode } \\
\hline $\mathrm{SBP}(\mathrm{mmHg})($ mean $(\mathrm{SD}))$ & $141(27)$ & $172(1.5)$ & $149(34)$ & $141(27)$ & $<0.001$ & $149(32)$ & $149(32)$ & 0.764 & 0 \\
\hline Heart rate (bpm) (mean (SD)) & $88.2(23.7)$ & $240(2.2)$ & $97.1(25.8)$ & $87.6(23.5)$ & $<0.001$ & $95(25)$ & $95(25)$ & 0.866 & 0 \\
\hline $\begin{array}{l}\text { Air-room pulse oximetry (\%) } \\
\text { (mean (SD)) }\end{array}$ & $92.2(6.5)$ & $350(3.1)$ & $87.8(9.7)$ & $92.6(6.1)$ & $<0.001$ & $88(8)$ & $88(10)$ & 0.736 & 0 \\
\hline \multicolumn{10}{|l|}{ Analytical data at ED } \\
\hline Glycemia (mg/dL) (median (SD)) & $146(82)$ & $184(1.6)$ & $184(95)$ & $143(80)$ & $<0.001$ & $173(78)$ & $176(104)$ & 0.589 & -1.7 \\
\hline $\begin{array}{l}\text { Creatinine }(\mathrm{mg} / \mathrm{dL}) \text { (median } \\
(\mathrm{SD}))\end{array}$ & $1.35(0.92)$ & $128(1.1)$ & $1.56(1.05)$ & $1.33(0.90)$ & $<0.001$ & $1.56(1.08)$ & $1.46(1.04)$ & 0.155 & +6.8 \\
\hline Hemoglobin (g/L) (median (SD)) & $121(43)$ & $96(9)$ & $120(27)$ & $121(44)$ & 0.755 & $121(29)$ & $123(68)$ & 0.475 & -1.6 \\
\hline $\begin{array}{l}\text { Potassium (mmol/L) (median } \\
\text { (SD)) }\end{array}$ & $4.6(5.4)$ & $684(6.1)$ & $4.9(5.5)$ & $4.6(5.4)$ & 0.124 & $4.9(6.4)$ & $4.5(2.1)$ & 0.160 & +8.9 \\
\hline Sodium (mmol/L) (median (SD)) & $137(13)$ & $185(1.7)$ & $137(7)$ & $137(13)$ & 0.457 & $137(8.1)$ & 139 (45.6) & 0.359 & -1.4 \\
\hline Troponin positive & $3578(57.6)$ & $4935(44.3)$ & $297(60.5)$ & $3281(57.3)$ & 0.185 & $189(57.4)$ & $171(60.0)$ & 0.577 & -4.3 \\
\hline \multicolumn{10}{|l|}{ Management at ED } \\
\hline $\begin{array}{l}\text { Receiving any intravenous } \\
\text { diuretic }\end{array}$ & $9440(84.7)$ & $3(0.0)$ & 660 (91.9) & $8780(84.2)$ & $<0.001$ & 460 (93.9) & $460(93.9)$ & 1.000 & 0 \\
\hline Receiving intravenous nitrates & $1517(13.6)$ & $1(0.0)$ & 351 (48.9) & $1166(11.2)$ & $<0.001$ & $218(44.5)$ & 217 (44.3) & 1.000 & +0.5 \\
\hline Receiving intravenous morphine & $682(6.1)$ & $1(0)$. & $267(37.2)$ & $415(4)$ & $<0.001$ & $143(29.2)$ & $147(30.0)$ & 0.834 & -2.7 \\
\hline $\begin{array}{l}\text { Receiving inotropics/ } \\
\text { vasopressors }\end{array}$ & $194(1.7)$ & $6(0.1)$ & $44(6.1)$ & $150(1.4)$ & $<0.001$ & $25(5.1)$ & $23(4.7)$ & 0.882 & +8.5 \\
\hline Admission at hospital & 8439 (75.7) & $6(0.1)$ & $690(96.1)$ & $7749(74.3)$ & $<0.001$ & $476(97.1)$ & $472(96.3)$ & 0.590 & +0.8 \\
\hline
\end{tabular}

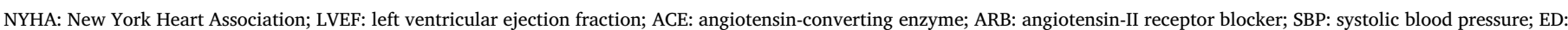
emergency department.

${ }^{a}$ RD denotes relative difference between mean of proportion of the NIV + group respect to the NIV- group.

patients, and AHF associated with ACS, our results suggest that NIV does not change 30-day all-cause mortality, although NIV may be associated with longer hospitalization. In view of our results which contradict those described in most RCT but are in line with those of the 3CPO trial, we believe that the use of NIV in the ED by emergency physicians to treat patients with AHF should be aimed at the amelioration of symptoms rather than to improvement of short-term survival.

Two main reasons could explain our results, which may be somewhat unexpected since NIV is widely used to treat AHF patients in the emergency arena. The first reason is that there is no a clear consensus in heart failure guidelines about when and in whom NIV should be applied. The NICE guidelines recommend to not routinely use NIV in people with AHF and cardiogenic APE, although it should to be considered for patients with respiratory distress [4]. This is different from the European Society of Cardiology (ESC) guidelines, which state that NIV should be considered in patients with respiratory distress (respiratory rate $>25$ breaths/min, SpO2 $<90 \%$ ) and started as soon as possible in order to decrease respiratory distress and reduce the rate of mechanical endotracheal intubation (class IIa recommedation, level of evidence B). Nonetheless, the ESC guidelines warn about NIV use in 


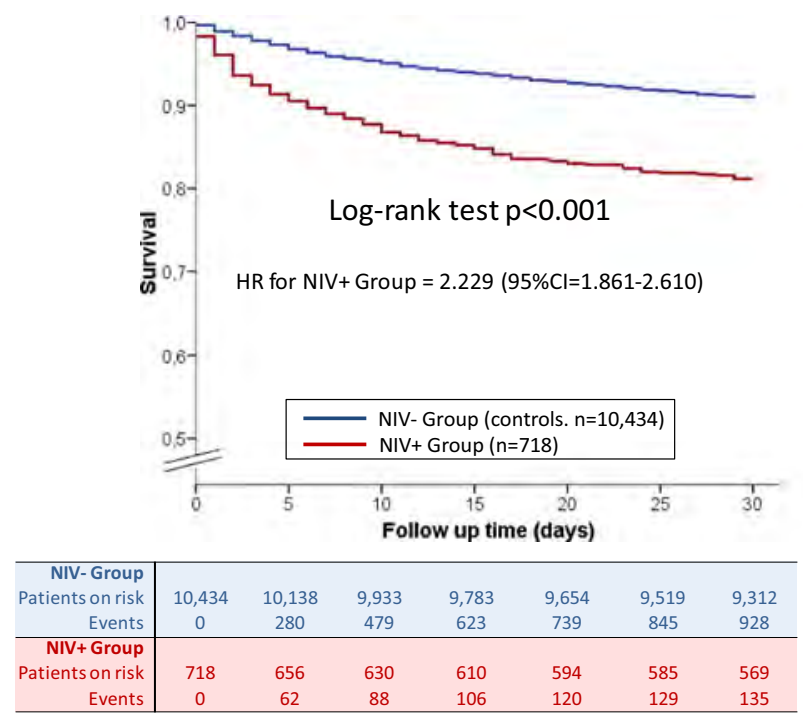

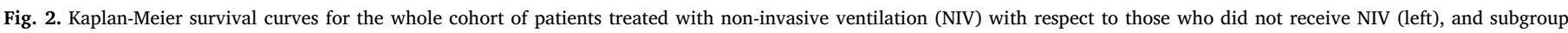

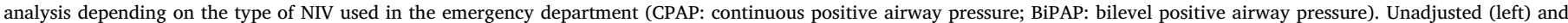
adjusted (right) proportional risk for patients that were treated with non-invasive ventilation (NIV) with respect to those who did not receive NIV.

hypotensive patients as it can decrease SBP, and they recognize that data on mortality are currently inconclusive [12]. Finally, the American College of Cardiology Foundation/American Heart Association guidelines do not make any specific statement regarding NIV use during an AHF decompensation [19]. In this scenario of conflicting guidelines, emergency physicians use NIV based on their own experience, clinical skills and subjective feelings rather than in well-defined patient profiles, likely leading to some heterogeneity in NIV use among ED physicians, and thereby influencing, in part, the results obtained in the NIVAHF Study.

The second potential explanation of our findings is the possibility that NIV has become a ceiling therapy for some of severely ill patients. This concept could likely explain the non-statistically significant trends homogenously pointing towards worse results with NIV use in our cohort, in which the mean patient age was 80 years and with many

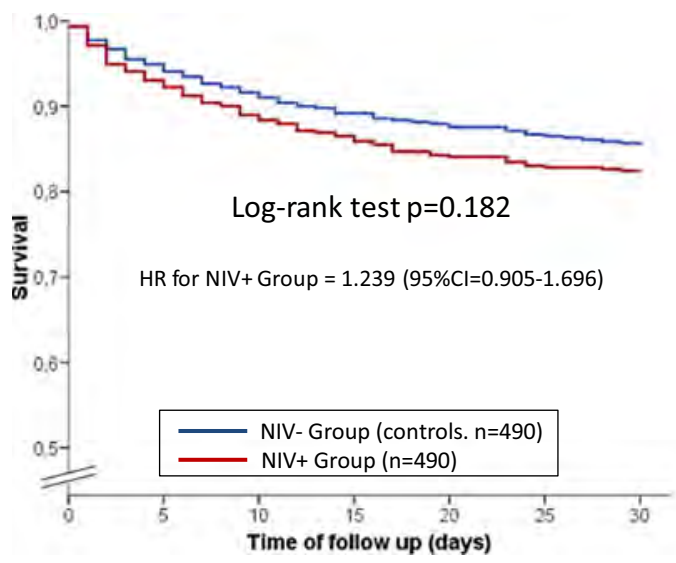

\begin{tabular}{r|ccccccc}
\hline NIV- Group & & & & & & & \\
Patients on risk & 490 & 465 & 449 & 436 & 427 & 421 & 409 \\
Events & 0 & 25 & 41 & 53 & 59 & 65 & 70 \\
\hline NIV+ Group & & & & & & & \\
Patients on risk & 490 & 456 & 436 & 422 & 409 & 403 & 391 \\
Events & 0 & 34 & 54 & 66 & 77 & 83 & 86 \\
\hline
\end{tabular}

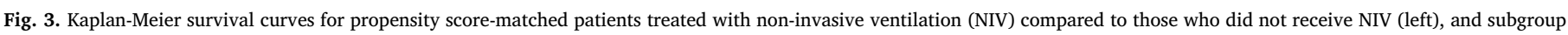
analysis depending on the type of NIV used in the emergency department (CPAP: continuous positive airway pressure; BiPAP: bilevel positive airway pressure).

patients presenting some functional limitations. In this scenario, the failure of NIV may not necessarily be followed by ETI and mechanical ventilation as some terminal patients could represent a cohort recognized as being in a "last hours situation". Unfortunately, we did not identify palliative care patients in our cohort. If supportive care patients with poor and extremely bad prognosis were included in the NIV + group, PS matching would not balance for this, thereby confounding our results.

Although these two facts may provide arguments against the relevance of our findings, we feel that it is of note that the mortality did not increase with NIV in the majority of our patients (not very eldery, had hypotension or had concomitant ACS). In addition, it should also be highlighted that the type of NIV was not related to outcomes. This is consistent with physiologic understanding of how these different strategies function. The clinical utility of a temporizing strategy allowing

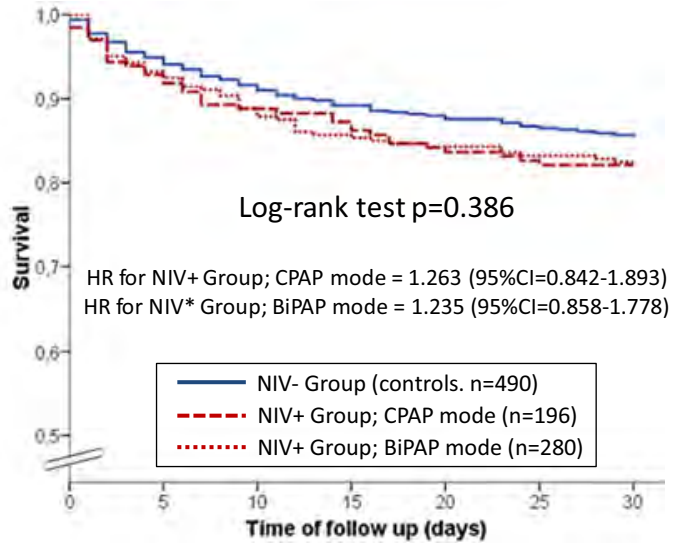

\begin{tabular}{r|ccccccc}
\hline NIV- Group & & & & & & & \\
Patients on risk & 490 & 465 & 449 & 436 & 427 & 421 & 409 \\
Events & 0 & 25 & 41 & 53 & 59 & 65 & 70 \\
\hline CPAP Group & & & & & & & \\
Patients on risk & 196 & 182 & 174 & 170 & 164 & 161 & 157 \\
Events & 0 & 14 & 22 & 25 & 31 & 34 & 35 \\
\hline BiPAP Group & & & & & & & \\
Patients on risk & 280 & 261 & 249 & 239 & 233 & 230 & 222 \\
Events & 0 & 19 & 31 & 40 & 44 & 47 & 49 \\
\hline
\end{tabular}




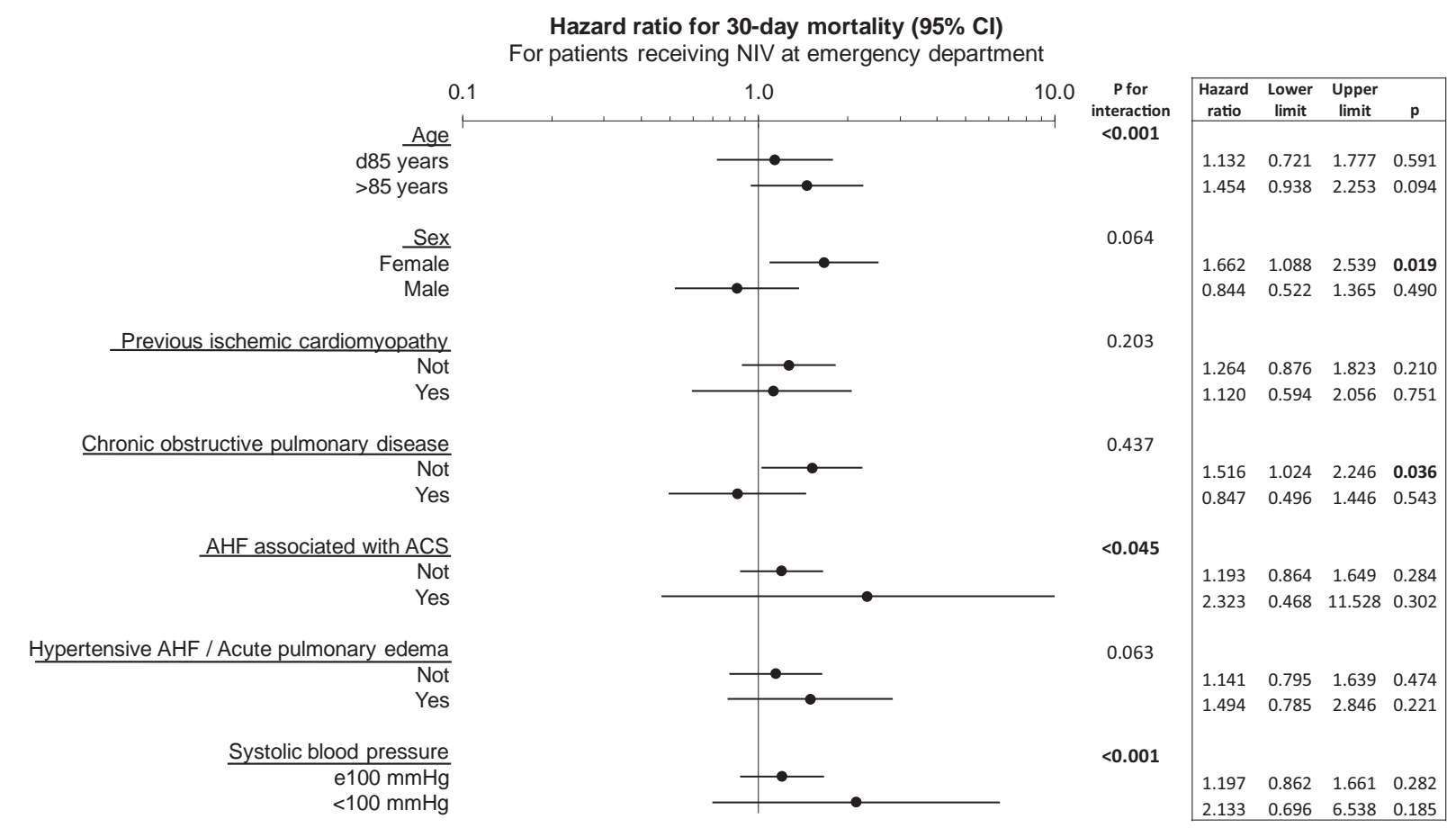

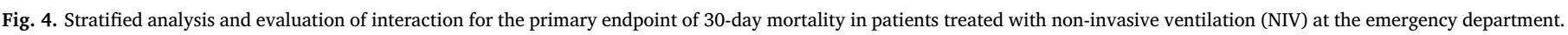
AHF: acute heart failure; ACS: acute coronary syndrome.

Table 2

Analysis of secondary endpoints in the two groups of patients matched by the propensity score.

\begin{tabular}{|c|c|c|c|c|}
\hline & $\begin{array}{l}\text { NIV + } \\
\text { group }\end{array}$ & $\begin{array}{l}\text { NIV- } \\
\text { group }\end{array}$ & $\begin{array}{l}\text { OR }(95 \% \mathrm{CI}) \text { for } \mathrm{NIV} \\
+ \text { group }\end{array}$ & $P$ value \\
\hline & (n (\%)) & (n (\%)) & & \\
\hline ICU admission & $29(5.9)$ & $17(3.5)$ & $1.754(0.951-3.236)$ & 0.072 \\
\hline Mechanical ventilation & $9(1.8)$ & $11(2.2)$ & $0.815(0.335-1.984)$ & 0.652 \\
\hline 3-day mortality & $29(5.9)$ & $22(4.5)$ & $1.338(0.758-2.364)$ & 0.316 \\
\hline 7-day mortality & $47(9.6)$ & $36(7.3)$ & $1.338(0.850-2.106)$ & 0.208 \\
\hline In-hospital mortality & $81(16.5)$ & $\begin{array}{l}61 \\
(12.4)\end{array}$ & $1.393(0.973-1.994)$ & 0.070 \\
\hline $\begin{array}{l}\text { Prolonged length of } \\
\text { stay ( }>7 \text { days) }\end{array}$ & $\begin{array}{l}261 \\
(53.9)\end{array}$ & $\begin{array}{l}217 \\
(44.7)\end{array}$ & $1.445(1.122-1.862)$ & 0.004 \\
\hline
\end{tabular}

alternative interventions (e.g., pharmacologic treatments) to potentially impart a beneficial effect before the patient required ETI and invasive ventilation did not change regardless of whether CPAP or BIPAP were used. It is important to recognize that NIV is not a destination therapy. It is used in an effort to avoid more invasive interventions that address severe respiratory compromise (e.g., ETI). As such, avoidance of ETI is an important outcome as patients receiving mechanical ventilation markedly increase resource utilization and are also at a much greater risk of aspiration and hospital-acquired pneumonia as compared to a non-intubated cohort $[20,21]$.

Special circumstances in which NIV use could imply increased risk merit discussion. There is general agreement that failure to improve the prognosis of patients with AHF during the last decades has been due, in part, to the lack of a definition of the different scenarios and different therapeutic approaches, as well as the unclear transition strategies undertaken in a paradigmatic multidisciplinary syndrome such as AHF [22-24]. Therefore, the targets of therapy for AHF should not only be to improve symptoms and hemodynamics, but also to preserve or improve renal function, prevent myocardial damage, modulate neurohumoral and inflammatory activation, manage other comorbidities, identify precipitants, and avoid secondary effects of treatments in particular subgroups of patients [24-26]. In the present study we identified that in patients with an ACS (different from STEMI, as this was an exclusion criteria), low SBP ( $<100 \mathrm{mmHg}$ ) and advanced age ( $>85$ years) NIV should probably be avoided. With respect to ACS, although mortality in those patients requiring NIV is increased respect the those not needing ventilatory support and decreased respect to those needing mechanical ventilation $[27,28]$, there are no RCT analyzing if the net effect of NIV in patients with ACS. On the other hand, although advanced age is associated with the use of NIV as limitation therapy, there is no clear cut-off for age in this sense. It seems reasonable that when applied to most elderly patients, NIV could be associated with worse outcomes due to its use as limitation therapy or related to the advanced age per se. However, regarding the latter, some recent data suggest that NIV success and mortality rates were similar between age groups [29]. Finally, with regard to hypotension, a recent study suggested that NIV could be effective in selected patients with cardiogenic shock, although when ventilatory support is needed, mechanical ventilation is the preferred option for most of these patients [30]. Again, in the absence of selection criteria for AHF patients with low SBP, our data agree with the ESC guidelines recommendations [12] of avoiding NIV use in the ED arena in hypotensive patients.

Our study has limitations. As an observational study, it is difficult to determine causality. The patients were not randomized and physicians could tend to reserve NIV for more severely ill cohorts. Propensity score matching attempted to adjust for known and measurable factors, but could not control for undocumented, unrecorded, or unknown variables and as such their consequences cannot be commented upon. Second, the impact of physician prescribing cannot be determined. It cannot be determined whether NIV + patients received the same therapeutic algorithmic care as NIV- patients because the individual time and drug used (e.g., $\mathrm{mg} / \mathrm{h}$ of nitroglycerin) were not recorded. It is possible that the presence of NIV masks decreased the frequency of sublingual nitroglycerin dosing, and that other factors resulted in therapeutic differences between NIV + and NIV-patients, possibly altering outcomes. The extent of this impact is unknown and was not measured. Third, we did not record whether the NIV was started by EMS in some patients, the delay in starting NIV after patient arrival to ED, or the total time 
that patients remained on NIV. Therefore, a particular subgroup of patients such as those with premature NIV weaning could have had a worse prognosis and possibly leaned the NIV + group towards a trend to worse results. Fourth, and similar to the latter limitation, aside from the mode (CPAP/BIPAP) of use ventilator parameters were not recorded, and in this scenario, it is again possible that ventilator conditions might not have been the most adequate in a subgroup of patients. Lastly, the NIV-AHF study only evaluated the effect of NIV use in the $\mathrm{ED}$, and it is possible that in some of our patients NIV could have been applied after they had been hospitalized.

We can conclude that NIV does not increase mortality in AHF except in the very eldery population ( $>85$ years), hypotensive patients (SBP $<100 \mathrm{mmHg}$ ), or in patients with AHF associated with ACS. It should generally not be used in these conditions. However, NIV may be appropriate in AHF when used to provide short-term symptom relief, or as a bridge to avoid ETI. Finally, since NIV does not ultimately have an impact on mortality, it should not be considered as definitive therapy but rather as a temporizing intervention.

Supplementary data to this article can be found online at https:// doi.org/10.1016/j.ejim.2018.03.008.

\section{Conflict of interests}

The authors state that they have no conflict of interests with the present work. The ICA-SEMES Research Group has received unrestricted support from Otsuka, Orion Pharma and Novartis. The present study has been designed, performed, analyzed and written exclusively by the authors independently of these pharmaceutical companies.

\section{Acknowledgments}

This study was partially supported by grants from the Instituto de Salud Carlos III(PI15/01019)supported with funds from the Spanish Ministry of Health and FEDER (PI15/01019 and PI15/00773) and La Marató de TV3 (2015/2510). The Emergencies: processes and pathologies research group of the IDIBAPS receives financial support from the Catalonian Government for Consolidated Groups of Investigation (GRC 2009/1385 and 2014/0313).

\section{References}

[1] Masip J, Roque M, Sánchez B, Fernández R, Subirana M, Expósito JA. Noninvasive ventilation in acute cardiogenic pulmonary edema: systematic review and metaanalysis. JAMA 2005;294:3124-30.

[2] Tallman TA, Peacock WF, Emerman CL, et al. Noninvasive ventilation outcomes in 2,430 acute decompensated heart failure patients: an adhere registry analysis. Acad Emerg Med 2008;15:355-62.

[3] Gray A, Goodacre S, Newby DE, et al. Noninvasive ventilation in acute cardiogenic pulmonary edema. N Engl J Med 2008;359:142-51.

[4] National Clinical Guideline Centre. Acute heart failure. diagnosing and managing acute heart failure in adults. NICE Clinical Guidelines. Vol. 187. London: National Institute for Health and Care Excellence (UK); 2014.

[5] Vital FM, Ladeira MT, Atallah AN. Non-invasive positive pressure ventilation (CPAP or bilevel NPPV) for cardiogenic pulmonary oedema. Cochrane Database Syst Rev 2013 May 31(5). http://dx.doi.org/10.1002/14651858.CD005351.pub3. (CD005351, Review).

[6] Miró O, Llorens P, Escalada X, et al. Prehospital emergency care of patients with acute heart failure in Spain: the SEMICA study (Emergency Medical Response Systems for Patients with Acute Heart Failure) SEMICA 1. Emergencias 2017;2017(29):223-30.

[7] Harjola P, Harjola VP. Can we do more for patients with acute heart failure before arrival at the hospital? Emergencias 2017;2017(29):221-2.
[8] Jacob J, Zorrilla J, Gené E, et al. Non-invasive ventilation in emergency departments in public hospitals in Catalonia. The VENUR-CAT study. Med Intensiva 2017 Jun 15. http://dx.doi.org/10.1016/j.medin.2017.05.002. (Epub ahead of print) pii: S0210-5691(17)30174-2.

[9] Miró Ò, Gil V, Müller C, et al. How does a clinical trial fit into the real world? The RELAX-AHF study population into the EAHFE registry. Clin Res Cardiol 2015;104:850-60.

[10] Carbajosa V, Martín-Sánchez FJ, Llorens P, et al. Factors associated with short stays for patients admitted with acute heart failure. Emergencias 2016;28:366-74.

[11] Miró Ò, Müller C, Martín-Sánchez FJ, et al. BETAWIN-AHF study: effect of betablocker withdrawal during acute decompensation in patients with chronic heart failure. Clin Res Cardiol 2016;105:1021-9.

[12] Ponikowski P, Voors AA, Anker SD, et al. 2016 ESC Guidelines for the diagnosis and treatment of acute and chronic heart failure: The Task Force for the diagnosis and treatment of acute and chronic heart failure of the European Society of Cardiology (ESC). Developed with the special contribution of the Heart Failure Association (HFA) of the ESC. Eur Heart J 2016;37:2129-200.

[13] Peacock WF, Nowak R, Christenson R, et al. Short-term mortality risk in emergency department acute heart failure. Acad Emerg Med 2011;18:947-58.

[14] Teixeira A, Parenica J, Park JJ, et al. Clinical presentation and outcome by age categories in acute heart failure: results from an international observational cohort. Eur J Heart Fail 2015;17:1114-23.

[15] Herrero-Puente P, Martín-Sánchez FJ, Fernández-Fernández M, et al. Differential clinical characteristics and outcome predictors of acute heart failure in elderly patients. Int J Cardiol 2012;155:81-6.

[16] Normand SL, Sykora K, Li P, Mamdani M, Rochon PA, Anderson GM. Readers guide to critical appraisal of cohort studies: 3 . Analytical strategies to reduce confounding. BMJ 2005;330:1021-3.

[17] Tannen RL, Weiner MG, Xie D. Use of primary care electronic medical record database in drug efficacy research on cardiovascular outcomes: comparison of database and randomised controlled trial findings. BMJ 2009;338:b81.

[18] Zhou Z, Rahme E, Abrahamowicz M, Pilote L. Survival bias associated with time-totreatment initiation in drug effectiveness evaluation: a comparison of methods. Am J Epidemiol 2005;162:1016-23.

[19] Yancy CW, Jessup M, Bozkurt B, et al. 2017 ACC/AHA/HFSA Focused Update on New Pharmacological Therapy for Heart Failure: An Update of the 2013 ACCF/AHA Guideline for the Management of Heart Failure: A Report of the American College of Cardiology/American Heart Association Task Force on Clinical Practice Guidelines and the Heart Failure Society of America. J Am Coll Cardiol 2017 Aug 8;70(6):776-803. http://dx.doi.org/10.1016/j.jacc.2017.04.025. Epub 2017 Apr 28.

[20] Masip J, Páez J, Merino M, et al. Risk factors for intubation as a guide for noninvasive ventilation in patients with severe acute cardiogenic pulmonary oedema. Intensive Care Med 2003;29:1921-8.

[21] Mebazaa A, Yilmaz MB, Levy P, et al. Recommendations on pre-hospital \& early hospital management of acute heart failure: a consensus paper from the Heart Failure Association of the European Society of Cardiology, the European Society of Emergency Medicine and the Society of Academic Emergency Medicine. Eur J Heart Fail 2015;17:544-58.

[22] De Luca L, Fonarow GC, Adams Jr. KF, et al. Acute heart failure syndromes: clinical scenarios and pathophysiologic targets for therapy. Heart Fail Rev 2007;12:97-104.

[23] Comín-Colet J, Enjuanes C, Lupón J, Cainzos-Achirica M, Badosa N, Verdú JM. Transitions of care between acute and chronic heart failure: critical steps in the design of a multidisciplinary care model for the prevention of rehospitalization. Rev Esp Cardiol 2016;69:951-61.

[24] Peacock WF. When and for how long do patients with acute heat failure have to be admitted? Emergencias 2016;28:363-5.

[25] Arrigo M, Gayat E, Parenica J, et al. Precipitating factors and 90-day outcome of acute heart failure: a report from the intercontinental GREAT registry. Eur J Heart Fail 2017;19:201-8.

[26] Aguirre Tejedo A, Miró O. Precipitating factors in acute heart failure: a review. Emergencias 2017;29:185-93.

[27] Metkus TS, Albaeni A, Chandra-Strobos N, Eid SM. Incidence and prognostic impact of respiratory support in patients with ST-segment elevation myocardial infarction. Am J Cardiol 2017;119:171-7.

[28] Tarvasmäki T, Harjola VP, Nieminen MS, et al. Acute heart failure with and without concomitant acute coronary syndromes: patient characteristics, management, and survival. J Card Fail 2014;20:723-30.

[29] Ozsancak Ugurlu A, Sidhom SS, Khodabandeh A, et al. Use and outcomes of noninvasive ventilation for acute respiratory failure in different age groups. Respir Care 2016 Jan;61(1):36-43.

[30] Hongisto M, Lassus J, Tarvasmaki T, et al. Use of noninvasive and invasive mechanical ventilation in cardiogenic shock: a prospective multicenter study. Int $\mathrm{J}$ Cardiol 2017;230:191-7. 